\title{
The Real-World Economic and Clinical Management of Adult Patients with Skin and Soft Tissue Infections (SSTIs) with Oritavancin: Data from Two Multicenter Observational Cohort Studies
}

\author{
Sandy Estrada ${ }^{1} \cdot$ Thomas P. Lodise $^{2} \cdot$ Glenn S. Tillotson ${ }^{3} \cdot$ Dino Delaportas $^{4}$
}

Published online: 25 June 2020

(c) The Author(s) 2020

\begin{abstract}
Background Oritavancin is a FDA-approved single-dose IV therapy for the treatment of acute bacterial skin and skin structure infections caused (or suspected to be caused) by certain Gram-positive pathogens, including methicillin-resistant Staphylococcus aureus (MRSA). Published data describing the outcomes of patients with skin and soft tissue infections (SSTIs) who received oritavancin beyond its pivotal phase III clinical trials are scant.

Objective The purpose of this report was to describe the results of two separate multicenter observational cohort studies that described the outcomes associated with two unique real-world usage patterns of oritavancin.

Methods The first cohort $(n=115)$ examined patients 18 years or older who were treated with oritavancin at three outpatient sites for SSTIs caused by suspected or confirmed Gram-positive pathogens, including MRSA, to avoid hospital admission. Patients were included if they had not been discharged from the inpatient setting within the previous $24 \mathrm{~h}$ and received their single-dose oritavancin treatment at a hospital-based outpatient infusion center. The primary outcomes measured were 30-day healthcare costs and admissions (all cause and infection related). The second cohort $(n=151)$ was a multicenter, retrospective chart review of adult patients who were discharged early from seven hospitals in 2015 on oritavancin for SSTIs. The primary outcome was readmission of patients within 30 days (all cause and infection related).

Results In cohort 1, 30-day mean healthcare costs were USD 3698. In the study of patients who started oritavancin in the outpatient setting, 7 patients $(6.1 \%)$ were admitted to hospital within 30 days of the index treatment, and 3 of those $(2.6 \%$ overall) were deemed to be due to an infection. In cohort 2, all-cause and infection-related 30-day readmission rates were $6.6 \%$ and $2.6 \%$, respectively, among patients who received oritavancin at hospital discharge.

Conclusions Findings from these studies suggest that oritavancin may be a potentially useful agent to avoid hospitalization or shorten hospital length of stay among appropriate SSTI patients. Future comparator studies are required to properly ascertain the outcomes and potential benefits associated with oritavancin relative to other commonly used antibiotics in patients with SSTIs.
\end{abstract}

\section{Introduction}

Digital features To view digital features for this article go to https://doi.org/10.6084/m9.figshare.12221690.

Dino Delaportas

drdelaportas@gmail.com

1 Lee Memorial Health System, Fort Myers, FL, USA

2 Albany College of Pharmacy and Health Sciences, Albany, NY, USA

3 GST Micro LLC, Henrico, VA, USA

4 Mon Health Medical Center, Mon General Hospital, $1200 \mathrm{~J}$ D Anderson Drive, Morgantown, WV, USA
The healthcare landscape is rapidly changing, largely due to changes introduced by the Affordable Care Act [1]. The payment of hospitals by both private and public payers is transitioning away from a fee-for-service model to a valuebased model where hospital payments are directly linked to the quality and efficiency of care that they provide [1, 2]. The value-based reimbursement model aligns well with the tenets of antimicrobial stewardship, which consists of systematic measurement and coordinated interventions that are designed to improve the use of antimicrobial medications with the goal of enhancing patient health outcomes 


\section{Key Points}

We describe two unique real-world usage patterns of oritavancin, a single-dose antibiotic with no therapeutic monitoring requirements, in patients with SSTIs.

Oritavancin was administered to stable SSTI patients in the outpatient setting to avoid costly hospitalization for IV antibiotic treatment; hospital admissions 30 days after treatment were low.

In hospitalized patients with SSTIs, oritavancin was used to facilitate early hospital discharge; infection-related 30-day hospital readmissions were low.

The findings suggest that oritavancin may assist in avoiding hospitalization and facilitating early discharge in select SSTI patients.

and decreasing unnecessary costs [3]. Effective in 2017, the Joint Commission on Hospital Accreditation required that all hospitals and nursing care centers have antimicrobial stewardship programs to facilitate the attainment of these healthcare goals [4].

As hospital reimbursement and antimicrobial stewardship programs are both increasingly tied to quality and efficiency of care, it is critical that healthcare institutions assess their approaches to care, especially for common and costly conditions such as skin and soft tissue infections (SSTIs). Skin and soft tissue infections are the seventh most common principal diagnosis in the United States for hospital admissions from the emergency department, resulting in over 600,000 hospital admissions per year [5]. The total spent on the treatment of patients with SSTIs has tripled since 2000, with yearly healthcare expenditure estimated to be nearly USD 14 billion in 2012 [6]. While inpatient care is commonplace for SSTI patients, data indicate that many hospitalized SSTI patients have zero or few uncontrolled comorbid conditions and no systemic signs and symptoms of infection, and that upwards of $85 \%$ are often solely admitted to receive IV antibiotics $[7,8]$. Because inpatient care for SSTI patients is 2-4 times more costly than outpatient care [9], healthcare institutions should identify appropriate patients for outpatient therapy and develop treatment strategies that can safely and effectively shift care from the inpatient to the outpatient setting among hospitalized patients [10].

One potential way to improve the efficiency of healthcare in patients with SSTIs is through the use of newer therapies such as oritavancin. Oritavancin is a FDA-approved single-dose IV therapy for the treatment of SSTIs caused (or suspected to be caused) by certain Gram-positive pathogens, including MRSA [11]. Oritavancin's broad spectrum of activity also includes vancomycin-resistant enterococci (VRE) [12], which may be relevant in complex SSTIs (e.g., polymicrobial infections and infections among patients with comorbidities such as diabetes mellitus) and are increasingly being identified in diabetic foot ulcers and wound infections [13]. Because it is a single-dose therapy, it can potentially be used to shift the site of care of stable patients with limited comorbidities who are admitted solely for the administration of IV antibiotics to the outpatient setting. There is also the potential to use oritavancin to facilitate the early discharge of a patient with a SSTI after their acute infection resolves. Despite its theoretical benefits, published data describing the real-world outcomes of patients who received oritavancin beyond its pivotal phase III clinical trials [14-16] are scant [17-19]. The purpose of this report was to describe the results of two separate multicenter observational cohort studies that examined the outcomes associated with two unique usage patterns of oritavancin. In the first cohort, oritavancin was used in the outpatient setting for patients with SSTIs as a measure to avoid hospitalization in suitable patients. In the second cohort, oritavancin was used to facilitate early hospital discharge in hospitalized patients with SSTIs.

\section{Methods}

\subsection{Cohort 1: Outpatient Use of Oritavancin to Avoid Admission}

This was a retrospective real-world study of patients 18 years or older who were treated in the outpatient setting with oritavancin for SSTIs caused by suspected or confirmed Gram-positive pathogens, including methicillin-resistant Staphylococcus aureus (MRSA). Patient charts from three hospital sites during the period $1 / 1 / 2015$ to $1 / 31 / 2016$ were analyzed. Consecutively treated patients were included if they had not been discharged from the inpatient setting within the previous $24 \mathrm{~h}$ and had received their single-dose oritavancin treatment at a hospital-based outpatient infusion center. The index date was defined as the date of oritavancin administration. Admissions were captured via an electronic health record system across all sites. Approval was obtained from the institutional review boards of Lee Memorial Health System (Fort Meyers, FL, USA) and Mon General Hospital (Morgantown, WV, USA) [20].

The primary assessment was healthcare resource utilization (HRU) from index treatment to 30 days post oritavancin administration. HRU included subsequent hospital admission, emergency department (ED) visits, antimicrobials and antimicrobial administration, diagnostics, and surgical procedures related to the infection. The total hospital costs from index treatment up to 30 days posttreatment were the sum of 
medication costs, the costs of all services patients received (including medication administration, ED visits, diagnostic tests, and surgical procedures), and the costs of subsequent hospitalization. The costs were calculated and presented in two ways: one was based on the mean of the unit costs; the other was based on the median of the unit costs. The wholesale acquisition cost (WAC) of oritavancin was used to estimate the medication costs [21]. The costs of healthcare resources were estimated using data from the Premier Hospital Database [22]. The cost of subsequent hospital admission was based on data from the Healthcare Cost and Utilization Project on the mean and median hospital costs of admission for SSTIs in 2013 [23]. The incidence rates of all-cause and infection-related hospital admissions were also determined.

\subsection{Cohort 2: Use of Oritavancin to Facilitate Early Discharge}

Seven hospitals implemented a strategy to discharge patients on oritavancin in order to avoid prolonged hospitalizations for IV antibiotic therapy. As a quality assurance measure, a multicenter, retrospective chart review of adult patients who were discharged from seven hospitals in 2015 while on oritavancin to treat a SSTI [24] was conducted.

The inclusion criterion was that the patient was receiving oritavancin upon hospital discharge to complete treatment of a SSTI. The exclusion criteria were the utilization of oritavancin upon discharge for a non-FDA-approved indication or treatment with oritavancin in the outpatient setting without prior hospitalization. Patient demographic characteristics, comorbidities, infection characteristics, and antibiotics received prior were summarized. All patients received antimicrobials (predominantly vancomycin) while they were inpatients prior to receiving oritavancin. The primary outcome was hospital readmissions within 30 days (all cause and infection related).

\section{Results}

\subsection{Cohort 1: Outpatient Use of Oritavancin to Avoid Admission}

In total, 115 patients met all inclusion criteria [20]. Patients had a mean age of 59.7 years and $40 \%$ were $>65$ years old; $93 \%$ of patients had at least one comorbidity, with hypertension being the most common (53\%), followed by hyperlipidemia $(20.9 \%)$ and diabetes $(22.6 \%)$. Additionally, $61.7 \%$ met the definition for obesity $\left(B M I \geq 30 \mathrm{~kg} / \mathrm{m}^{2}\right)$. Sixtynine $(60 \%)$ patients had received antibiotics prior to oritavancin, including 21 with IV/IM antibiotics and 63 with oral antibiotics, some of which covered both Gram-positive and Gram-negative pathogens. In the majority of patients (81.7\%), the infection was first diagnosed in a physician's office, and the overwhelming majority of the patients (93.9\%) received oritavancin treatment from a hospitalowned outpatient infusion center. Of the 56 patients with baseline culture results, MRSA was identified in 27 (48.2\%). The infection types were cellulitis (70\%), wound (19\%), and major cutaneous abscess (11\%). Most infections were on the leg $(51 \%)$ or foot $(21 \%)$.

The estimated hospital costs (mean and median) for services provided to patients were calculated using data from the 2013 Healthcare Cost and Utilization Project, and are shown in Table 1. Total costs for outpatient treatment of an SSTI patient with oritavancin, including follow-up costs, were estimated to be USD 3698 (mean) and USD 3341 (median). Six (5.2\%) patients received diagnostic testing as part of their index treatment, which included ultrasonography, X-ray, and CT scan. Twenty-six patients (22.6\%) received at least one surgical procedure during index treatment. Surgical procedures included incision and drainage (8 patients), surgical debridement (18 patients), negative pressure wound therapy (1 patient), and other (1 patient).

Eleven patients $(9.6 \%)$ received antibiotics within 30 days post index treatment. Reasons for antibiotic use included inadequate treatment response $(n=1)$, relapse of the same infection $(n=2)$, new Gram-positive infection $(n=2)$, the primary infection was both Gram positive and Gram negative $(n=4)$, and standard of care or hospital protocol $(n=3)$. Seven patients $(6.1 \%)$ were admitted to hospital within 30 days of the index treatment. One admission was due to a vascular issue rather than an infection, and required surgery. Three of these admissions (2.6\% overall) were due to an infection, with Gram-negative bacteria identified,

Table 1 Estimated 2018-2019 hospital costs (in USD) for services provided to patients with SSTIs [6, 22]

\begin{tabular}{lcc}
\hline Service & $\begin{array}{l}\text { Mean hospital } \\
\text { costs (USD) }\end{array}$ & $\begin{array}{c}\text { Median hospi- } \\
\text { tal costs (USD) }\end{array}$ \\
\hline Oritavancin (WAC) & 2900.00 & 2900.00 \\
Drug administration (3 h) & 514.81 & 238.02 \\
Ultrasonography & 269.14 & 204.48 \\
X-ray & 82.48 & 68.97 \\
CT scan & 180.88 & 127.12 \\
Incision and drainage & 101.02 & 78.39 \\
Surgical debridement & 299.46 & 165.00 \\
Negative pressure wound therapy & 182.72 & 161.09 \\
ED visit & & \\
Level IV & 260.67 & 226.69 \\
Level V & 377.03 & 331.33 \\
Hospital admission for SSTI & 6823.00 & 5074.00 \\
\hline
\end{tabular}

$E D$ emergency department, SSTI skin and soft tissue infection, WAC wholesale acquisition cost 
and required antibiotic therapy. None of the three patients who were hospitalized with a Gram-negative infection had received antibiotics with Gram-negative activity previously. One $(0.9 \%)$ patient visited the emergency department within 30 days of index treatment and received antibiotics but was not hospitalized.

\subsection{Cohort 2: Use of Oritavancin to Facilitate Early Discharge}

Overall, 151 patients were included [25]. The mean age was 56 years (range 19-90) and most were female (65\%). Diabetes was reported in $38 \%$ of patients, and $5 \%$ of patients were intravenous drug users. The most frequent infection types were cellulitis (75\%), wound (11\%), and major cutaneous abscess (10\%). Among patients with available culture results ( $n=78$ ), MRSA (32 patients) was the most common pathogen. Gram-negative bacteria were recovered from a culture in 9 patients. Reflective of real-world hospital clinical practices, the antibiotics administered to patients upon initial presentation varied, and some had Gram-negative coverage. Most patients received vancomycin (78\%) prior to discharge with oritavancin. Other frequently used antibiotics prior to oritavancin were clindamycin $(11 \%)$, ceftaroline (9\%), daptomycin (7\%), and linezolid (5\%). Seven patients received concomitant antimicrobials with oritavancin for the treatment of Gram-negative organisms.

Ten patients $(6.6 \%)$ were readmitted at 30 days, and 4 of those readmissions (2.6\%) were attributable to infection. Of those 4,2 patients were admitted to treat a Gram-negative infection, and 2 patients were admitted for abscess drainage ( 1 was found to be MSSA and the other was culture negative). The two patients with Gram-negative infections received inappropriate antibiotic therapy for Gram-negative pathogens prior to the administration of oritavancin. None of the patients with a non-infection-related hospital admission required additional antibiotic therapy after oritavancin.

\section{Discussion}

Given the frequency of SSTIs and associated healthcare costs [5, 26], management of patients with SSTIs should be explored as a quality improvement initiative by US healthcare systems. Currently, many patients with SSTIs who present to the ED with demonstrable SSTIs are subsequently hospitalized and receive multidose IV antibiotics, even in the setting of mild or no systemic signs of infection $[7,8]$. Data also indicate that patients hospitalized for SSTIs often remain in the hospital after their acute infection resolves [27]. The burden of this practice on the healthcare system is substantial. Hospital stays for patients with SSTIs range from 3.5 to 10 days [28, 29], resulting in hospital costs of between USD 5000 and USD 18,014 in 2015 [30]. Recent data also show that $9.8 \%$ of patients with cellulitis were readmitted in US hospitals; age and insurance status were the main risk factors. Moreover, overall annual costs associated with nonelective readmissions due to SSTIs within 30 days of a cellulitis discharge in the US were estimated to total USD 114.4 million (95\% CI: USD 106.8-122.0 million) [31].

While a variety of components comprise the cost of inpatient care, it is due in large part to room and board costs $[7,32]$. Data indicate that a proportion of SSTI patients may be able to receive their care in the outpatient setting or may be discharged from the hospital sooner with continued antibiotic therapy without compromising clinical outcomes $[27,33]$. Though an early transition from intravenous to oral therapy may be one strategy, treatment challenges still exist. Most oral therapies are dosed several times a day, which may result in undue toxicity or poor patient adherence. One study found that patient adherence to oral SSTI treatment postdischarge was low (57\%), which resulted in a poor 30-day clinical response for nearly half of patients [34].

One way to potentially improve the efficiency of healthcare delivery for SSTI patients is through the use of oritavancin. As it is administered in a single dose and does not require therapeutic monitoring [11], it has been hypothesized that oritavancin may be an ideal treatment in the ambulatory setting, particularly in stable patients with moderate to severe SSTIs. When evaluating the viability of antibiotic agents such as oritavancin for outpatient use, there are several important considerations. First, the agent must be efficacious. The candidate outpatient SSTI therapy must confer an early, demonstrable response that is sustained over time until resolution. The response rates also must be similar for the various types of patients that present with SSTIs. Second, it must have a highly favorable safety profile. Adverse events must only occur at a low frequency and must be of a mild, self-limiting nature. Third, therapy cannot result in an excessive number of hospital admissions, as this defeats the intent of shifting care to the outpatient setting. Fourth, it is highly desirable to establish its efficacy and safety in the outpatient setting in a multicenter, randomized, double-blind, comparative clinical trial, as this is the gold standard study design for establishing the clinical viability of new agents. Finally, there should ideally be supportive evidence for its use in real-world effectiveness studies that substantiate proposed treatment pathways.

To date, the results of phase III clinical trials have indicated that a single dose of oritavancin has comparable efficacy to twice-daily intravenous vancomycin for 7-10 days in the outpatient setting [14-16]. Oritavancin was generally 
well tolerated, and very few patients in the oritavancin group required subsequent care in the inpatient setting post outpatient treatment [16]. Although there are challenges to directly extrapolating real-world effectiveness outcomes from phase III trials, these data support the use of oritavancin in the outpatient setting. However, it is important to note that patients in the oritavancin group were required to return to the outpatient setting daily to receive placebo doses to maintain blinding. Therefore, questions about the real-world implications of a single dose of oritavancin administered in the outpatient setting remain, as the effect of additional touchpoints with a healthcare provider on the reported outcomes are unknown.

There were two notable findings across the two multicenter real-world evidence cohort studies included in this report that address potential data gaps with employing oritavancin as a treatment option to shift care from the inpatient to the outpatient setting. Overall, the incidences of 30-day hospital admissions/readmissions post receipt of oritavancin were nearly identical and low in the two cohorts. Seven patients $(6.1 \%)$ had a 30-day hospital admission and 3 (2.6\% overall) were deemed to be infection related in the study of patients who started oritavancin in the outpatient setting (cohort 1). Similarly, all-cause and infection-related 30 -day readmission rates were $6.6 \%$ and $2.6 \%$, respectively, among patients who received oritavancin at hospital discharge (cohort 2). In both cases, the rates of hospital admission post oritavancin treatment were lower than nationally reported rates of overall (20\%) and infection-related (10\%) readmissions for patients with SSTIs [31]. It should be noted that not every admission/readmission may have been captured, as a small number of patients may have been referred to another health system.

Second, healthcare resource use data suggest that oritavancin may be a cost-saving approach to treating appropriate SSTI patients in the outpatient setting. The cost of outpatient treatment with oritavancin, inclusive of follow-up costs, was estimated to be $\sim$ USD 3500 in cohort 1 . This is considerably lower than the costs associated with inpatient treatment with other IV antibiotics. Even for SSTI patients with no or just one comorbidity who have no systemic signs and symptoms of infection, the mean inpatient cost is estimated to be USD 6000 [7]. Thus, there is a potential cost saving of USD 3000 with oritavancin relative to current inpatient care, assuming no admissions in the oritavancin group.

Several things should be noted when interpreting the findings. As this was a retrospective real-world analysis, it is subject to all the limitations of observational real-world studies. Although these studies were not comparative, the study population is representative of patients with skin infections treated in the outpatient setting at the included hospitals, but it may not represent typical outpatient populations at other sites. For example, nearly two-thirds of the population were obese. Cost data were not available from the hospitals where this study was conducted. Therefore, the costs were derived and do not reflect the true costs to the institutions included in this analysis. Unknown admissions could have also occurred outside the system. Finally, a comparator treatment group was not available, and future studies are needed to fully understand outcomes associated with oritavancin relative to other antibiotics commonly used in patients with SSTIs.

\section{Conclusion}

In summary, it is important to develop new therapies and treatment approaches that maintain or improve outcomes at the lowest overall cost in the new value-based hospital payment model. The concept of quality of care at lowest cost is congruent with the goals of antibiotic stewardship programs, which have a dual responsibility to optimize clinical outcomes and minimize healthcare costs without adversely impacting quality of care. Findings from this report suggest that oritavancin may be a potentially useful agent to avoid hospitalization or shorten hospital LOS among appropriate SSTI patients while saving institutional costs. Future comparator studies are required to properly ascertain the outcomes and potential benefits associated with oritavancin and other long-acting single-dose lipoglycopeptides relative to other antibiotics commonly used in patients with SSTIs.

Acknowledgements The authors thank Nicolette Theriault for her editorial assistance. The authors wish to thank their clinical colleagues at Lee Memorial Hospital, Fort Myers, FL; Mon General Hospital, Morgantown, WV; United Hospital Center, Bridgeport, WV; and Fairmont Regional Medical Center, Fairmont, WV.

\section{Compliance with Ethical Standards}

Funding The study and manuscript preparation were not funded. This manuscript is part of a supplement wholly funded by Melinta Therapeutics.

Data Availability All data generated or analyzed during this study are included in this published article (and its supplementary information files).

Conflict of Interest TPL is a consultant and speaker for Melinta Therapeutics. GT has received consulting fees from Melinta Therapeutics, Summit plc, Spero Therapeutics, Shionogi Inc., and KBP Biosciences. DD is a speaker for Melinta Therapeutics and received a grant from The Medicines Company. SE has no disclosures. 
Ethics Approval IRB approval was given by Lee Health System (Fort Myers, FL) and Mons General Hospital (Morgantown, WV), and the study has been performed in accordance with the ethical standards of the Declaration of Helsinki.

Authorship All authors had a role in study design and in conceiving and writing the manuscript. According to the guidelines of the International Committee of Medical Journal Editors (ICMJE, https://www. icmje.org), all authors met the criteria for authorship, and no deserving authors have been omitted.

Open Access This article is licensed under a Creative Commons Attribution-NonCommercial 4.0 International License, which permits any non-commercial use, sharing, adaptation, distribution and reproduction in any medium or format, as long as you give appropriate credit to the original author(s) and the source, provide a link to the Creative Commons licence, and indicate if changes were made. The images or other third party material in this article are included in the article's Creative Commons licence, unless indicated otherwise in a credit line to the material. If material is not included in the article's Creative Commons licence and your intended use is not permitted by statutory regulation or exceeds the permitted use, you will need to obtain permission directly from the copyright holder. To view a copy of this licence, visit http://creativecommons.org/licenses/by-nc/4.0/.

\section{References}

1. Burwell SM. Setting value-based payment goals: HHS efforts to improve US health care. N Engl J Med. 2015;372(10):897-9.

2. Centers for Medicare and Medicaid Services. Hospital Readmission Reduction Program 2018 [updated 12/04/2018 5:03 PM]. https://www.cms.gov/Medicare/Quality-Initiatives-Patient-Asses sment-Instruments/Value-Based-Programs/HRRP/HospitalReadmission-Reduction-Program.html.

3. Barlam TF, Cosgrove SE, Abbo LM, MacDougall C, Schuetz AN, Septimus EJ, et al. Implementing an antibiotic stewardship program: guidelines by the Infectious Diseases Society of America and the Society for Healthcare Epidemiology of America. Clin Infect Dis. 2016;62(10):e51-77.

4. Joint Commission on Hospital Accreditation. Approved: new antimicrobial stewardship standard. Jt Comm Perspect. 2016;36(7).

5. US Department of Health and Human Services. Healthcare Cost Utilization Project HCUPnet-principal diagnosis only restrict tables to non-neonatal, non-maternal discharges, outcomes by 197 skin and subcutaneous tissue infections 2019. https://hcupn et.qa.s-3.net.

6. Lee GC, Boyd NK, Lawson KA, Frei CR. PIN100: incidence and cost of skin and soft tissue infections in the United States. Val Health. 2015;18(3):A245.

7. Lodise TP, Fan W, Sulham KA. Hospital admission patterns in adult patients with skin and soft tissue infections: identification of potentially avoidable hospital admissions through a retrospective database analysis. Hosp Pract. 2015;43(3):137-43.

8. Talan DA, Salhi BA, Moran GJ, Mower WR, Hsieh YH, Krishnadasan A, et al. Factors associated with decision to hospitalize emergency department patients with skin and soft tissue infection. West J Emerg Med. 2015;16(1):89-97.

9. Khachatryan A, Ektare V, Xue M, Dunne M, Johnson KE, Stephens JM. Reducing total health care costs by shifting to outpatient (OP) settings of care for the management of Gram+ acute bacterial skin and skin structure infections (ABSSSI). Val Health. 2013;16(3):A203.
10. Whittaker C, Nhan E, Ganapathiraju P, Garrett S, Reilly J, Travedi $M$. Expediting discharge in acute bacterial skin and skin structure infections: a clinical and economic comparison between Vancomycin and Oritavancin in hospitalized patients. Open Forum Infect Dis. 2018;5(Suppl 1):S118.

11. Melinta Therapeutics, Inc. Orbactiv ${ }^{\circledR}$ (oritavancin): full prescribing information (package insert). New Haven: Melinta Therapeutics, Inc.; 2018.

12. Brade KD, Rybak JM, Rybak MJ. Oritavancin: a new lipoglycopeptide antibiotic in the treatment of gram-positive infections. Infect Dis Ther. 2016;5(1):1-15.

13. O'Driscoll T, Crank CW. Vancomycin-resistant enterococcal infections: epidemiology, clinical manifestations, and optimal management. Infect Drug Resist. 2015;8:217-30.

14. Corey GR, Good S, Jiang H, Moeck G, Wikler M, Green S, et al. Single-dose oritavancin versus 7-10 days of vancomycin in the treatment of gram-positive acute bacterial skin and skin structure infections: the SOLO II noninferiority study. Clin Infect Dis. 2015;60(2):254-62.

15. Corey GR, Kabler H, Mehra P, Gupta S, Overcash JS, Porwal A, et al. Single-dose oritavancin in the treatment of acute bacterial skin infections. N Engl J Med. 2014;370(23):2180-90.

16. Lodise TP, Redell M, Armstrong SO, Sulham KA, Corey GR. Efficacy and safety of oritavancin relative to vancomycin for patients with acute bacterial skin and skin structure infections (ABSSSI) in the outpatient setting: results from the SOLO clinical trials. Open Forum Infect Dis. 2017;4(1):ofw274.

17. Co D, Roebuck L, VanLandingham J. Evaluation of oritavancin use at a community hospital. Hosp Pharm. 2018;53(4):272-6.

18. Redell M, Moeck G, Lucasti C, Durso S, Kennedy C, Fusaro K, et al. A real-world patient registry for oritavancin demonstrates efficacy and safety consistent with the phase 3 SOLO program. Open Forum Infect Dis. 2018;5(6):ofy051.

19. Whittaker C, Nhan E, Ganapathiraju P, Garrett S, Reilly J, Trivedi $M$. Expediting discharge in acute bacterial skin and skin structure infections: a clinical and economic comparison between vancomycin and oritavancin. Presented at: ID Week; 2018 Oct 3-7; San Francisco, CA, USA.

20. Turner E, Estrada S, Galli A, Armstrong S, Delaportas D. Treatment of acute bacterial skin and skin structure infections (ABSSSI) in the outpatient setting: clinical and economic outcomes from a real-world multi-center study of oritavancin. Poster No.1855. ASHP Conference for Pharmacy Leaders. Chicago, IL, USA. October 17-18, 2016. https://melintamedicalinformation .com/Media/Publications/ORBACTIV/ASHP\%202016/Turne r\%20EJ\%20Poster\%20241855\%20ASHP\%202016.pdf.

21. Wolters Kluwer Clinical Drug Information, Inc. Medi-Span Price Rx: Oritavancin. https://pricerx.medispan.com/.

22. Premier Inc. Premier Healthcare Database White Paper: Data that informs and performs. Charlotte: Premier Inc.; 2018.

23. Agency for Healthcare Research and Quality. Healthcare Cost Utilization Project: Clinical Classifications Software (CCS) for ICD-9-CM. 2019. https://www.hcup-us.ahrq.gov/toolssoftware/ ccs/ccs.jsp.

24. US Food and Drug Administration. Guidance for industry. Acute bacterial skin and skin structure infections: developing drugs for treatment. Silver Spring: Center for Drug Evaluation and Research; 2013.

25. Estrada S, Delaportas D, El-Imad B, Stanley J. Real-world experience utilizing oritavancin to discharge patients with ABSSSI (poster 20). Presented at: Making a Difference in Infectious Diseases (MAD-ID); 2016 May 5-7; Orlando, FL, USA.

26. Lee G, Boyd N, Lawson K, Frei C. Incidence and cost of skin and soft tissue infections in the United States. Val Health. 2015;18(3):A245. 
27. Dryden M, Saeed K, Townsend R, Winnard C, Bourne S, Parker $\mathrm{N}$, et al. Antibiotic stewardship and early discharge from hospital: impact of a structured approach to antimicrobial management. J Antimicrob Chemother. 2012;67(9):2289-96.

28. Suaya JA, Mera RM, Cassidy A, O'Hara P, Amrine-Madsen H, Burstin S, et al. Incidence and cost of hospitalizations associated with Staphylococcus aureus skin and soft tissue infections in the United States from 2001 through 2009. BMC Infect Dis. 2014;14:296.

29. Kaye KS, Patel DA, Stephens JM, Khachatryan A, Patel A, Johnson K. Rising United States hospital admissions for acute bacterial skin and skin structure infections: recent trends and economic impact. PLoS ONE. 2015;10(11):e0143276.

30. Ektare V, Khachatryan A, Xue M, Dunne M, Johnson K, Stephens $\mathrm{J}$. Assessing the economic value of avoiding hospital admissions by shifting the management of gram+ acute bacterial skin and skin-structure infections to an outpatient care setting. J Med Econ. 2015;18(12):1092-101.
31. Fisher JM, Feng JY, Tan SY, Mostaghimi A. Analysis of readmissions following hospitalization for cellulitis in the United States. JAMA Dermatol. 2019;155(6):720-3.

32. Sulham K, LaPensee K, Fan W, Lodise TP. Severity and costs of acute bacterial skin and skin structure infections by treatment setting: an application of the Eron classification to a real-world database. Val Health. 2014;17(3):A282.

33. Nathwani D, Eckmann C, Lawson W, Stephens JM, Macahilig C, Solem CT, et al. Pan-European early switch/early discharge opportunities exist for hospitalized patients with methicillin-resistant Staphylococcus aureus complicated skin and soft tissue infections. Clin Microbiol Infect. 2014;20(10):993-1000.

34. Eells SJ, Nguyen M, Jung J, Macias-Gil R, May L, Miller LG. Relationship between adherence to oral antibiotics and postdischarge clinical outcomes among patients hospitalized with Staphylococcus aureus skin infections. Antimicrob Agents Chemother. 2016;60(5):2941-8. 